\title{
A Retrospective Risk Analysis of Factors Associated with Prolonged Hospitalization in Adult Patients Undergoing Non-surgical Treatment for Appendiceal Abscess
}

\author{
Masatsugu Hiraki ${ }^{1}{ }^{*}$, Toshiya Tanaka ${ }^{1}, K$ Kohei $\operatorname{Yamada}^{1}$, Eiji Sadashima ${ }^{2}$, Tatsuya Manabe ${ }^{3}$, \\ Kenji Kitahara ${ }^{1}$, Hirokazu Noshiro ${ }^{3}$ \\ ${ }^{1}$ Department of Surgery, Saga Medical Center Koseikan, Saga, Japan \\ ${ }^{2}$ Life Science Research Institute, Saga Medical Center Koseikan, Saga, Japan \\ ${ }^{3}$ Department of Surgery, Saga University Faculty of Medicine, Saga, Japan
}

Email address:

masatsuguhiraki@hotmail.com (M. Hiraki)

${ }^{*}$ Corresponding author

\section{To cite this article:}

Masatsugu Hiraki, Toshiya Tanaka, Kohei Yamada, Eiji Sadashima, Tatsuya Manabe, Kenji Kitahara, Hirokazu Noshiro. A Retrospective Risk Analysis of Factors Associated with Prolonged Hospitalization in Adult Patients Undergoing Non-surgical Treatment for Appendiceal Abscess. Journal of Surgery. Vol. 8, No. 1, 2020, pp. 16-21. doi: 10.11648/j.js.20200801.14

Received: January 10, 2020; Accepted: January 21, 2020; Published: February 7, 2020

\begin{abstract}
Background: The aim of the present study was to investigate risk factors and predictive factors for prolonged hospitalization in adults undergoing non-surgical treatment for appendiceal abscess. Patients and Methods: A retrospective study was conducted of consecutive patients who underwent treatment following a diagnosis of appendicitis in a single institution. Between January 2008 and May 2019, 756 adult patients who were diagnosed with acute appendicitis, 101 of these patients $(13.4 \%)$ had an appendiceal abscess and were treated as inpatients. The treatments of these patients were as follows: non-surgical treatment alone $(n=45)$, emergency operation $(n=50)$ and conversion to emergency operation due to failure of the initial conservative therapy $(n=6)$. The present study analyzed the 45 patients managed with non-surgical treatment alone. The patients were divided, based on the median length of the hospital stay (nine day), into the shorter hospital stay and longer hospital stay groups. The risk factors and predictive factors for prolonged hospitalization were examined in the two groups. Results: Twenty-four patients were classified into the shorter hospital stay group and 21 were classified into the longer hospital stay group. A univariate analysis demonstrated that the body temperature on post-admission days $2(P=0.012)$ and $3(P=0.008)$, were significantly associated with the length of hospital stay. A multivariate logistic regression analysis using that the body temperature on post-admission day 3 tended to be associated with prolonged hospitalization (odds ratio $=8.574,95 \%$ confidence interval $=0.973-75.525 ; \mathrm{P}=0.053$ ). The cut-off value of the body temperature on day 3 , determined by an ROC curve analysis, was $37.05^{\circ} \mathrm{C}$. This cut-off value showed $66.7 \%$ accuracy, $57.1 \%$ sensitivity and $75.0 \%$ specificity. Conclusion: The body temperature on post-admission day 3 might be a potential risk factor and predictive marker for prolonged hospitalization in patients who receive non-surgical treatment for appendiceal abscess.
\end{abstract}

Keywords: Appendiceal Abscess, Appendicular Abscess, Non-Surgical Treatment, Appendicitis, Interval Appendectomy

\section{Introduction}

Appendicitis is one of the most common surgical emergencies and appendectomy has been established as a standard treatment for appendicitis. However, 3.3-9.7\% of patients experience complicated appendicitis with abscess formation at the initial diagnosis [1-3]. If an emergency operation is performed in such cases, extended organ resection, including ileocecal resection or conversion from laparoscopic surgery to laparotomy might be necessary, and postoperative complications (e.g., wound infection, abdominal abscess, or intestinal adhesion) may occur [4-9]. Thus, elective interval appendectomy following initial nonsurgical treatment, including antibiotic therapy and/or percutaneous drainage has recently come to be widely 
accepted instead of the traditional emergency operation [4, 5]. However, non-surgical treatment with the aim of performing elective interval appendectomy sometimes might be unsuccessful and can cause prolonged hospitalization and impair the patient's quality of life $[2,10]$. Thus, it is important to understand the risk factors and predictive factors for prolonged hospitalization in patients who receive nonsurgical treatment for appendiceal abscess.

The aim of the present study was to identify the risk factors and predictive factors for prolonged hospitalization in adults undergoing non-surgical treatment for appendiceal abscess.

\section{Methods}

\subsection{Patients}

A retrospective study was conducted for consecutive patients diagnosed with appendicitis who were treated in a single institution at the Department of Surgery, Saga Medical Center Koseikan, between January 2008 and May 2019. During this period, 756 adult patients were diagnosed with acute appendicitis in our department of surgery. A consort diagram is shown in Figure 1. Among all of the patients, 101 patients $(13.4 \%)$ patients presented with an appendiceal abscess and were treated as inpatients. Among these patients, 51 patients were initially managed with non-surgical treatment, whereas the remaining 50 patients underwent an emergency operation. Among the patients initially managed with non-surgical treatment, conversion to an emergency operation due to failure of the conservative therapy was required in six cases. The present study analyzed the cases of 45 patients for whom non-surgical treatment was successful. The medical records of all patients were reviewed in detail. All patients and their families were informed about the treatment and gave their informed broad consent for this study. Appendicitis with appendiceal abscess was confirmed by a physical examination, blood test and contrast abdominal computed tomography (CT). If there were no signs of peritonitis, sepsis, shock, and/or organ failure, non-surgical treatment was considered. During the non-surgical treatment, if signs of peritonitis and/or a worsening of symptoms were observed, conversion to emergency operation was considered. Abscess drainage was decided by the doctor-incharge depending on the patient's condition and/or a worsening of symptoms and laboratory data. If there was no worsening of symptoms and the patient was able to eat solid food, then the patient was discharged from hospital with/without treatment with oral antibiotics. The following data were collected from the prospectively maintained comprehensive database or medical records: sex, age, body mass index, previous history, current smoking habit, physical examination, CT parameters (identification of appendix, appendicolith, abscess diameter), types of antibiotics used for treatment, blood test results, body temperature, percutaneous abscess drainage and hospital stay. Continuous variables were expressed as the median and range (minimum, maximum). For the purpose of this study, the patients managed with non-surgical treatment alone were divided, based on median length of the hospital stay, into the shorter hospital stay and longer hospital stay groups. The cut-off value for the length of hospital stay was nine days. The medical ethics committee of Saga Medical Center Koseikan reviewed and approved this study design (permission number: 19-07-01-04).

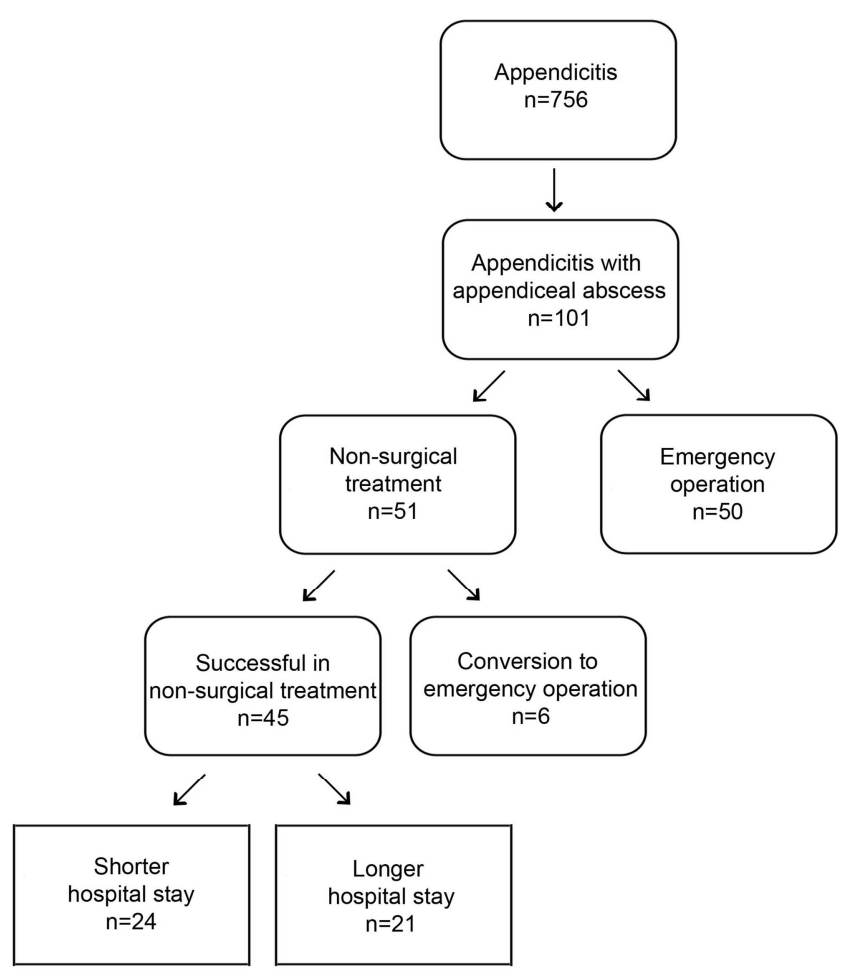

Figure 1. A consort diagram of this study.

\subsection{Statistical Analyses}

For the univariate analyses, continuous variables were compared between the groups by the Mann-Whitney U test. while Fisher's exact test was used for the comparison of binary variables. Receiver operation characteristics (ROC) analyses were performed and the area under the curve (AUC) was calculated to examine the capability of potential markers. Continuous variables were converted into binary variables based on optimal cut-off values determined by ROC analyses. A multiple logistic regression analysis was performed to identify factors that were independently associated with a prolonged hospital stay. The data were expressed as odds ratios (ORs) with 95\% confidence intervals (CIs). $\mathrm{P}$ values of $<0.05$ were considered to indicate statistical significance. All analyses were performed using SPSS version 25.0. (IBM Corp, Armonk, NY, USA).

\section{Results}

The study population included $28(62 \%)$ male patients and $17(38 \%)$ female patients. The age of the patients ranged from 15 to 94 years. In all patients, a peri-appendiceal 
abscess measuring 14.0 to $110.0 \mathrm{~mm}$ in size was detected by enhanced computed tomography.

Table 1 shows the results of the univariate analysis of patient-related factors. Twenty-four and 21 patients were classified into the shorter and longer hospital stay groups, respectively.

Table 1. A univariate analysis of patient -related factors.

\begin{tabular}{|c|c|c|c|}
\hline & Shorter & Longer & \multirow{2}{*}{$P$-value } \\
\hline & hospital stay $(n=24)$ & hospital stay $(n=21)$ & \\
\hline Gender (male: female) & $16: 8$ & $12: 9$ & 0.552 \\
\hline Age (years: median (min, $\max )$ ) & $52.0(21,88)$ & $59(15,94)$ & 0.991 \\
\hline Body mass index $\left(\mathrm{kg} / \mathrm{m}^{2}:\right.$ median $\left.(\min , \max )\right)$ & $23.2(17.0,31.2)$ & $22.8(20.5,41.9)$ & 0.601 \\
\hline \multicolumn{4}{|l|}{ Previous history } \\
\hline Cardiovascular disease (yes: no) & $2: 22$ & $5: 16$ & 0.225 \\
\hline Diabetes mellitus (yes: no) & $1: 23$ & $1: 20$ & 1.000 \\
\hline Current Smoking (yes: no) & $6: 18$ & $5: 16$ & 1.000 \\
\hline Pre-hospital symptom duration (days: median [min, max]) & $3[0,25]$ & $2[0,19]$ & 0.296 \\
\hline \multicolumn{4}{|l|}{ Physical examination } \\
\hline Tenderness (yes: no) & $24: 0$ & $20: 1$ & 0.467 \\
\hline Muscular defense (yes: no) & $4: 20$ & $6: 15$ & 0.476 \\
\hline Rebound tenderness (yes: no) & $11: 13$ & $9: 12$ & 1.000 \\
\hline \multicolumn{4}{|l|}{$\mathrm{CT}$ parameters } \\
\hline Appendicolith (yes: no) & $7: 17$ & $6: 15$ & 1.000 \\
\hline Being able to identify the appendix (yes: no) & $5: 19$ & $3: 18$ & 0.750 \\
\hline Abscess size (mm: median (min, max)) & $33.5(14.0,110.0)$ & $31.0(17.0,65.0)$ & 0.641 \\
\hline \multicolumn{4}{|l|}{ Antibiotics } \\
\hline TAZ/PIPC based & 11 & 6 & \multirow{5}{*}{0.454} \\
\hline $\mathrm{SBT} / \mathrm{ABPC}$ based & 5 & 7 & \\
\hline CMZ based & 3 & 5 & \\
\hline LVFZ based & 4 & 3 & \\
\hline Other & 1 & 0 & \\
\hline Hemoglobin (g/dl: median (min, $\max )$ ) & $13.8(10.7,15.8)$ & $14.4(10.3,18.0)$ & 0.119 \\
\hline Albumin (g/dl: median (min, max)) & $3.8(2.7,4.6)$ & $3.8(2.5,5.0)$ & 0.971 \\
\hline \multicolumn{4}{|l|}{ White blood cells $(/ \mu \mathrm{l}$ : median (min, max $))$} \\
\hline Pretreatment & $11900(5100,21000)$ & $14400(4400,22500)$ & 0.357 \\
\hline Post admission day 1 & $9600(4000,18700)$ & $12700(2900,18700)$ & 0.289 \\
\hline Post admission day 3 & $6450(3800,12400)$ & $9350(3200,18200)$ & 0.065 \\
\hline \multicolumn{4}{|l|}{ C-reactive protein (mg/dl: median (min, max)) } \\
\hline Pretreatment & $12.5(0.2,28.6)$ & $12.3(0.2,28.3)$ & 0.716 \\
\hline Post admission day 1 & $12.9(0.7,23.6)$ & $13.7(5.1,28.6)$ & 0.196 \\
\hline Post admission day 3 & $9.1(0.3,22.1)$ & $11.8(1.8,26.9)$ & 0.208 \\
\hline \multicolumn{4}{|l|}{ Body temperature $\left({ }^{\circ} \mathrm{C}\right.$ : median (min, $\left.\left.\max \right)\right)$} \\
\hline Pretreatment & $37.4(36.3,39.3)$ & $37.6(36.4,39.8)$ & 0.316 \\
\hline Post admission day 1 & $37.4(36.4,39.3)$ & $37.7(36.5,39.2)$ & 0.305 \\
\hline Post admission day 2 & $37.0(36.1,38.2)$ & $37.3(36.8,39.3)$ & 0.012 \\
\hline Post admission day 3 & $36.9(36.1,37.4)$ & $37.1(36.5,39.3)$ & 0.008 \\
\hline Percutaneous abscess drainage (yes: no) & $1: 23$ & $6: 15$ & 0.039 \\
\hline Antibiotics change due to disease progression (yes: no) & $1: 23$ & 11: 10 & $<0.001$ \\
\hline Hospital stay (days) & $7.5(4,9)$ & $15(10,23)$ & $<0.001$ \\
\hline
\end{tabular}

A univariate analysis demonstrated the pre-hospital symptom duration, the presence of abdominal symptoms (tenderness, muscular defense and rebound tenderness), the CT findings of the appendix (the detection of an appendicolith, being able to identify the appendix on CT images, and the abscess size), the type of antibiotics administered, the white blood cell count and the C-reactive protein level to not be associated with the length of the hospital stay. In contrast, body temperature on postadmission days $2(P=0.012)$ and $3 \quad(P=0.008)$, were significantly associated with the length of hospital stay (Figure 2A, 2B).
Percutaneous abscess drainage $(P=0.039)$ and change of antibiotics due to disease progression $(P<0001)$ were performed significantly more frequently in the longer hospital stay group. A multivariate logistic regression analysis that included the body temperature on postadmission days 2 and 3 was performed to identify factors independently associated with a prolonged hospital stay. The analysis revealed that the body temperature on postadmission day 3 tended to be associated with a prolonged hospital stay (odds ratio $=8.574, \quad 95 \%$ confidence interval $=0.973-75.525 ; \mathrm{P}=0.053)$ (Table 2$)$. 
A

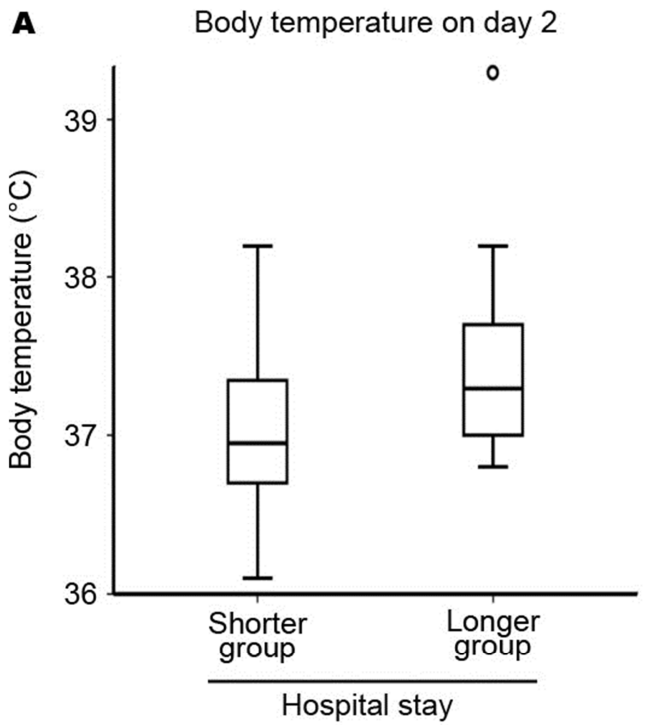

C

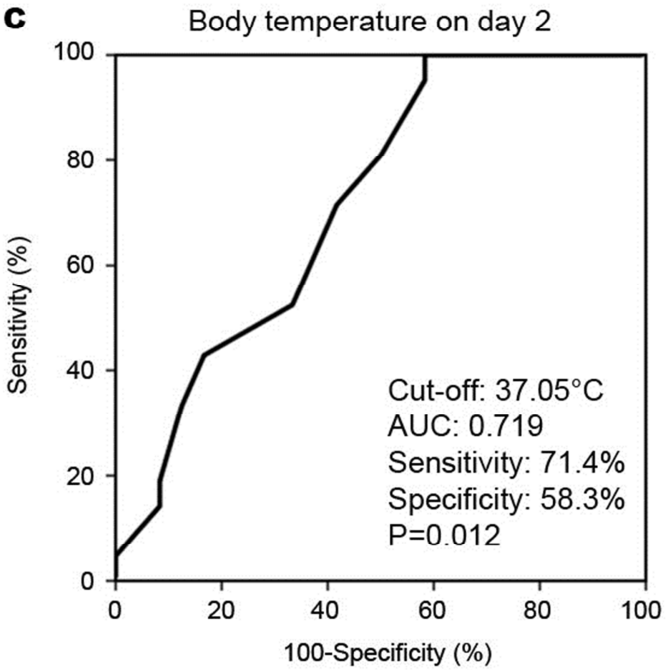

B

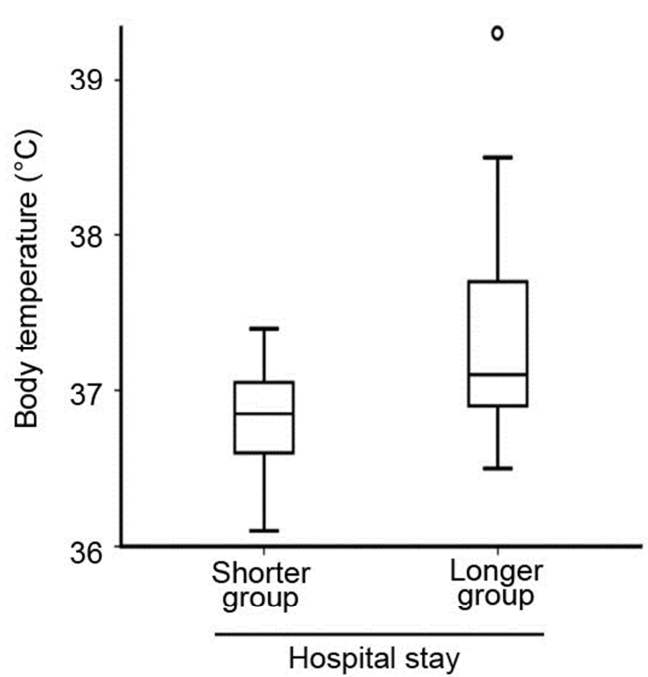

D

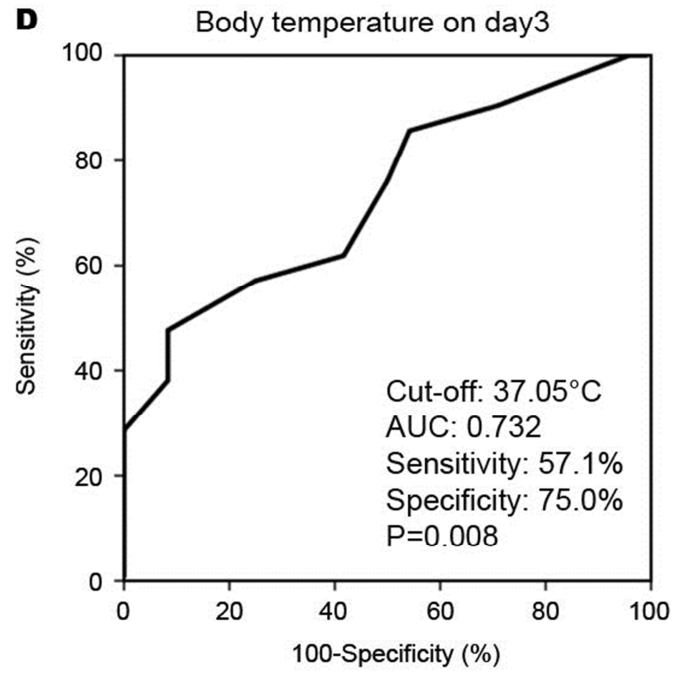

Figure 2. Relationship between body temperature after admission and hospital stay.

Table 2. A multivariate analysis of patient-related factors.

\begin{tabular}{lll}
\hline \multirow{2}{*}{ Parameters } & multivariate analysis & \\
\cline { 2 - 3 } & OR (95\% C. I.) & P-value \\
\hline Body temperature on post admission day 2 & $1.560(0.300-8.124)$ & 0.597 \\
Body temperature on post admission day 3 & $8.574(0.973-75.525)$ & 0.053 \\
\hline
\end{tabular}

ROC curve analyses were then performed to determine the cut-off values of body temperature on post-admission day 2 and 3 that best predicted a prolonged hospital day. A body temperature of $37.05^{\circ} \mathrm{C}$ on post-admission days 2 and 3 was determined based on the area under the curve (AUC) (Figure $2 \mathrm{C}, 2 \mathrm{D})$. The ROC analysis demonstrated that this value showed $66.4 \%$ accuracy, $71.4 \%$ sensitivity, and $58.3 \%$ specificity on post-admission day 2 and $66.7 \%$ accuracy, $57.1 \%$ sensitivity, and $75.0 \%$ specificity on post-admission day 3 .

\section{Discussion}

A recent study reported that elective interval appendectomy for appendiceal abscess after non-surgical treatment is widely accepted strategy [4, 5]. Non-surgical treatment for appendiceal abscess is reported to be associated with a relatively highly success rate of $74-76 \%[2,11]$. However, prolonged hospitalization following the failure of the non-surgical treatment can still be an issue [1, 2, 10-13]. Even though effective antibiotic regimens and procedures for abscess drainage have been established, the failure of nonsurgical treatment due to a delaying in changing antibiotics or introducing abscess drainage may lead to prolonged hospitalization, causing an impaired quality of life for the patient and resulting in increased medical costs. The best strategy for appendiceal abscess in adults is still debatable. Even if non-surgical treatment is successful and allows the avoidance of conversion to emergency surgery in most cases, prolonged hospitalization itself can still be a disadvantage for 
the patient. Thus, the identification of risk factors and predictive factors for prolonged hospitalization due to appendiceal abscess in patients undergoing non-surgical treatment is important for optimizing the treatment strategy.

The risk factors and predictive factors for the failure of non-surgical treatment resulting in an emergency operation has been previously reported [2]. However, this kind of study mainly only aims to examine the factor for the failure of nonsurgical treatment followed by conversion to emergency operation. Our study focused on patients with appendiceal abscess who were managed with non-surgical treatment alone. Cases in which conversion to an emergency operation was required were excluded because only 6 patients required conversion to surgery, and this number was considered too small to analyze. In addition - in a broad sense-it was also considered that cases involving prolonged hospitalization for non-surgical treatment should be considered as cases of nonsurgical treatment failure.

In this study, a higher body temperature on post admission day 3 was significantly associated with prolonged hospitalization in the univariate analysis and a borderline significant association was observed in the multivariate analysis. In this series, antibiotics were changed and/or percutaneous abscess drainage was performed due to disease progression in $52 \%(11 / 21)$ and $29 \%(6 / 21)$ of the patients in the longer hospitalization group, respectively. Among these cases, the treatment strategy was changed on post-admission day 2-12 (median: day 4) and day 0-12 (median: day 4), respectively. The ROC curve analysis demonstrated that the cut-off value for the body temperature on post-admission day 3 to predict prolonged hospitalization was $37.05^{\circ} \mathrm{C}$. Taken together, these results indicate that changing the treatment strategy when a patient presents a body temperature of $>37.05^{\circ} \mathrm{C}$ on post-admission day 3 has the potential to be shorten the hospital stay.

Previous reports have demonstrated that the abscess size can be an important factor for treatment results in appendicitis and diverticulitis. Regarding the risk factors for conversion to an emergency operation following unsuccessful initial non-surgical treatment, Sadakari et al. reported that an abscess size of $>40 \mathrm{~mm}$ could predict failure of non-surgical treatment resulting in an emergency operation in patients with appendiceal abscess [2]. Kim et al. analyzed unfavorable clinical outcomes of acute right colonic diverticulitis and revealed that an abscess of $>4 \mathrm{~cm}$ was a significant predictor of a prolonged hospital stay [14]. In addition, Mali et al. reported that diverticulitis with abscess was mostly treated with antibiotics alone if the size was $<40$ $\mathrm{mm}$, with a high treatment success rate $(87 \%)$, and found that the abscess size was correlated with emergency surgery or prolonged hospitalization [15]. As for our study, if an abscess size of $4 \mathrm{~cm}$ was used as the cut off value in our study, abscesses of $>4 \mathrm{~cm}$ in diameter were seen in $48 \%$ of the patients with a shorter hospital stay and $42 \%$ of the patients with a longer hospital stay; this difference was not statistically significant. The reasons for this discrepancy are not clear; however, it may have been due to the small number of patients. Thus, further analyses will be needed in future studies.

The present study was associated with some limitations. First, the study was conducted at a single institution with a relatively small number of subjects. Thus, further studies will be needed to confirm the utility of body temperature on postadmission day 3 as a predictive marker for prolonged hospitalization in patients with appendiceal abscess who are managed with conservative therapy. In addition, the diagnostic accuracy still needs to be improved. Therefore, another novel risk factor or predictive factor for prolonged hospitalization should be identified in patients managed with non-surgical treatment for appendiceal abscess.

In conclusion, the body temperature on post-admission day 3 might have potential application as a predictive marker for prolonged hospitalization for patients with appendiceal abscess who are managed by conservative therapy.

\section{Author Contributions}

M Hiraki and $\mathrm{T}$ Toshiya mainly designed this study. M Hiraki, T Tanaka, K Yamada, K Kitahara treated the patients. M Hiraki T Tanaka, and Yamada collected data. M Hiraki and E Sadashima (specialist in statistics) analyzed the data. $M$ Hiraki, T Tanaka, T Manabe and H Noshiro interpreted the result and wrote the manuscript. All the authors met the following criteria. The authors made substantial contributions to the conception and design, and/or acquisition of data, and/or analysis and interpretation of data, participated in drafting the article or revising it critically for important intellectual content, and gave final approval of the version to be published.

\section{Conflicts of Interest}

The authors declare no conflict of interest in association with the present study.

\section{References}

[1] Helling TS, Soltys DF, Seals S. Operative versus nonoperative management in the care of patients with complicated appendicitis. Am J Surg 2017; 214: 1195-1200.

[2] Sadakari Y, Date S, Murakami S et al. Prediction of negative outcomes in non-surgical treatment for appendiceal abscess in adults. J Anus Rectum Colon 2018; 2: 59-65.

[3] de Jonge J, Bolmers MDM, Musters GD et al. Predictors for interval appendectomy in non-operatively treated complicated appendicitis. Int J Colorectal Dis 2019; 34: 1325-1332.

[4] Di Saverio S, Birindelli A, Kelly MD et al. WSES Jerusalem guidelines for diagnosis and treatment of acute appendicitis. World J Emerg Surg 2016; 11: 34.

[5] Gorter RR, Eker HH, Gorter-Stam MA et al. Diagnosis and management of acute appendicitis. EAES consensus development conference 2015. Surg Endosc 2016; 30: 46684690 . 
[6] Simillis C, Symeonides P, Shorthouse AJ, Tekkis PP. A metaanalysis comparing conservative treatment versus acute appendectomy for complicated appendicitis (abscess or phlegmon). Surgery 2010; 147: 818-829.

[7] Shekarriz S, Keck T, Kujath $P$ et al. Comparison of conservative versus surgical therapy for acute appendicitis with abscess in five German hospitals. Int J Colorectal Dis 2019; 34: 649-655.

[8] Mentula P, Sammalkorpi H, Leppaniemi A. Laparoscopic Surgery or Conservative Treatment for Appendiceal Abscess in Adults? A Randomized Controlled Trial. Ann Surg 2015; 262: $237-242$

[9] Quah GS, Eslick GD, Cox MR. Laparoscopic appendicectomy is superior to open surgery for complicated appendicitis. Surg Endosc 2019; 33: 2072-2082.

[10] Nimmagadda N, Matsushima K, Piccinini A et al. Complicated appendicitis: Immediate operation or trial of nonoperative management? Am J Surg 2019; 217: 713-717.
[11] Young KA, Neuhaus NM, Fluck $M$ et al. Outcomes of complicated appendicitis: Is conservative management as smooth as it seems? Am J Surg 2018; 215: 586-592.

[12] Watanabe R, Otsuji A, Nakamura Y et al. Superior outcomes (but at higher costs) of non-operative management with interval appendectomy over immediate surgery in appendicitis with abscess: Results from a large adult population cohort. Asian J Endosc Surg 2019.

[13] Chen CL, Chao HC, Kong MS, Chen SY. Risk Factors for Prolonged Hospitalization in Pediatric Appendicitis Patients with Medical Treatment. Pediatr Neonatol 2017; 58: 223 228.

[14] Kim DH, Kim HJ, Jang SK et al. CT Predictors of Unfavorable Clinical Outcomes of Acute Right Colonic Diverticulitis. AJR Am J Roentgenol 2017; 209: 1263-1271.

[15] Mali J, Mentula P, Leppaniemi A, Sallinen V. Determinants of treatment and outcomes of diverticular abscesses. World $\mathrm{J}$ Emerg Surg 2019; 14: 31. 